\title{
Efficacy and safety of crizotinib in patients with anaplastic lymphoma kinase-positive advanced- stage non-small-cell lung cancer
}

This article was published in the following Dove Press journal: Cancer Management and Research

\author{
Ahmed Mohieldin ${ }^{1,2}$ \\ Ayman Rasmy 1,3,4 \\ Mohamed Ashour ${ }^{2,5}$ \\ Muath Al-Nassar ${ }^{6}$ \\ Rola H Ali ${ }^{7,8}$ \\ Fahad G El-Enezi ${ }^{6}$ \\ 'Medical Oncology, Zagazig \\ University Hospitals, Zagazig, Egypt; \\ ${ }^{2}$ Medical Oncology Department, \\ Sheikha Badriya Alsabah Centre, \\ Kuwait Cancer Control Center, \\ Shuwaikh, Kuwait; ${ }^{3}$ Medical Oncology \\ Department, King Saud Medical \\ City, Riyadh, Saudi Arabia; ${ }^{4}$ Medical \\ Oncology Department, King Fahad \\ Specialist Hospital, Dammam, Saudi \\ Arabia; ${ }^{5} \mathrm{Clinical}$ Oncology, Al-Azhar \\ University, Cairo, Egypt; ${ }^{6}$ Thoracic \\ Oncology - Sheikha Badriya \\ Alsabah Centre, Kuwait Cancer \\ Control Center, Shuwaikh, Kuwait; \\ ${ }^{7}$ Department of Pathology, Faculty \\ of Medicine, Kuwait University, Safat, \\ Kuwait; ${ }^{8}$ Molecular Laboratory, Kuwait \\ Cancer Control Center, Shuwaikh, \\ Kuwait
}

Introduction: Lung cancer is the leading cause of cancer mortality worldwide, despite advances in management, especially with targeted agents and immunotherapy. Numerous oncogenes have been identified that control the growth of these malignancies. Anaplastic lymphoma kinase (ALK) is a tyrosine kinase that develops distorted functioning as a result of chromosomal rearrangement. Crizotinib, a tyrosine kinase inhibitor (TKI), was approved by the Food and Drug Administration (FDA) in 2011 for the treatment of advanced ALK-positive non-small-cell lung cancer (NSCLC).

Patients and methods: In this chart review, we compiled data from two cancer hospitals in Kuwait and Saudi Arabia which were collected from patients with advanced NSCLC treated between January 2013 and September 2017 with crizotinib after diagnosed with ALK-positive disease. Crizotinib $250 \mathrm{mg}$ BID was given orally with/without food intake. We assessed overall survival (OS), objective response rate (ORR), progression-free survival (PFS), duration of the response, and dose reduction/cessation.

Results: De-identified data from 38 subjects were compiled. Their median age was 53 years, $65.8 \%$ were male, the 1 -year OS was $88 \%$, and the PFS was 16.5 months. Two cases $(5.3 \%)$ had a complete response (CR), while 17 (44.7\%) had a partial response (PR). Side effects of grade III/IV occurred, including elevated transaminase levels, diarrhea, and prolonged QT intervals, in $8 \%$ patients, with dose reduction in six patients (15.8\%).

Conclusion: In NSCLC, crizotinib is a viable treatment option with good response and tolerable toxicity for patients with ALK-positive advanced disease.

Keywords: non-small-cell lung cancer, anaplastic lymphoma kinase gene, crizotinib, overall survival, progression-free survival

\section{Introduction}

Although lung malignancies are the second most common type of cancers, they are consistently the main cause of malignancy-related mortality in the world (in about $14 \%$ of all cases). The lung cancer incidence estimated by the American Society of Clinical Oncology (ASCO) for USA in 2018 is 234,030 new cases (121,680 in men and 112,350 in women), with mortality estimated at 154,050 (including 83,550 men and 70,500 women). ${ }^{1}$ During the past 15 years, the approach to treat non-small-cell lung cancer (NSCLC) cases has changed from "chemo-for-all" to the use of a "tailored medicine" strategy. Oncologists now have identified that at least one driver change, including gene mutation, rearrangement, or amplification, occurs in $50 \%$ of the cases of metastatic lung adenocarcinoma., ${ }^{2,3}$
Correspondence: Ayman Rasmy Medical Oncology, Zagazig University,

Zagazig, Sharkia, Egypt

Tel +20 I I I 4535000

Email ay_rasmy@yahoo.com 
Chromosomal rearrangement has a role in the activation of tyrosine kinase inhibitor (TKI; anaplastic lymphoma kinase $[\mathrm{ALK}]$ ), which leads to the expression of "EML4ALK" as an "oncogenic fusion kinase." 4,5 Additional ALK gene changes similarly happen in anaplastic large-cell lymphoma, ${ }^{6}$ pediatric neuroblastoma, ${ }^{7}$ and inflammatory myofibroblastic tumors. ${ }^{8}$

For patients having advanced NSCLC with ALK-positive subtypes, new treatment options have been developed using ALK gene rearrangement studies confirmed by "Food and Drug Administration (FDA)-approved tests". Currently, the FDA has granted different levels of approval for the various small molecule TKIs developed for the management of NSCLC with different ALK rearrangements, in that these agents confer different levels of clinical improvement in comparison to standard chemotherapy.

Crizotinib as a TKI has been approved for the management of cancers utilizing tyrosine kinases such as ALK, ${ }^{5}$ MET, ${ }^{9}$ or $\mathrm{ROS}^{10}{ }^{10}$ since August 2011, when the FDA recognized it as a first-generation ALK inhibitor for treating such cases. ${ }^{10}$ After 2 years, regular approval for crizotinib was granted by the FDA.

In the cases where ALK rearrangements occurred, leading to disease recurrence or progression after crizotinib treatment, the second- and third-generation oral ALK inhibitors (such as ceritinib, alectinib, or brigatinib) have been approved. ${ }^{11-13}$ These newer inhibitors have more potent effects against the different EML4-ALK resistance point mutations.

Based on the ALEX trial, in November 2017, alectinib was approved by the FDA for the management of NSCLC in ALK-positive cases. ${ }^{14}$ In February 2018, the FDA announced the auditing of another application (for lorlatinib, a thirdgeneration ALK inhibitor) regarding the ability to resensitize cancer cells to crizotinib. ${ }^{15}$

\section{Patients and methods}

This chart review "retrospective" study focused on the outcomes of advanced NSCLC "stage IIIB and IV" diagnosed at the oncology departments of the Kuwait Cancer Control Center (KCCC) in Kuwait and the King Fahad Specialist Hospital (KFSH) in Saudi Arabia between January 2013 and September 2017. All cancer diagnoses were confirmed by surgical pathology from tissues obtained from either primary lung lesions or metastatic sites. Each diagnosis of adenocarcinoma thus was confirmed by immunohistochemistry (IHC) by an expert pathologist. Evaluations of EGFR mutations, ALK rearrangements, and ROS1 rearrangements were carried out to identify potentially targetable drive alterations. The ALK FISH gene rearrangement evaluation was performed in all patients centrally using CE-marked Vysis LSI ALK Break Apart Rearrangement Probe Kits (Abbott Laboratories, Abbott Park, IL, USA) that detect ALK locus rearrangements on chromosome $2 \mathrm{p} 23$.

Patient characteristics, such as age; sex; comorbidities; initial symptoms; metastatic site; prior therapy including surgery, chemotherapy, crizotinib, and local treatment by radiotherapy (RT); response to therapy; pattern of failure; time to progression (TTP); and survival outcome, were documented. All cases had initial staging by "computed tomography (CT) and/or magnetic resonance imaging (MRI) scans" prior to starting crizotinib. Platinum combination chemotherapy was allowed as the first-line treatment. Palliative RT was given at any time to control localized pain or for hemostatic purposes using three-dimensional (3D) conformal RT.

All patients were given crizotinib either as the firstline treatment and/or subsequent treatment upon failure of platinum-based chemotherapy or as a switch maintenance after achieving the maximum response on platinum-based chemotherapy. Crizotinib was recommended as standard dose "250 mg orally every 12 hours with or without meal" until treatment failure or intolerable therapy-related side effects. Dose modification was allowed according to patient tolerance.

Outpatient treatment was carried out with regular evaluation during visits by clinical and laboratory assessment including complete blood counts ( $\mathrm{CBCs}$ ), liver and renal function tests, bone panels, and electrocardiograms. Evaluations of the response were carried out by repeating the CT and/or MRI scans by 3-4-month interval. The response status was recorded according to RECIST 1.0 criteria (Response Evaluation Criteria in Solid Tumors). In the case of solitary recurrence, metastasectomy, stereotactic, and/or gamma knife therapy were performed.

\section{Statistical analyses}

Statistical evaluations were carried out by SPSS software version 23.0 (IBM Corporation, Armonk, NY, USA).

All the following are considered as per standard guidelines: response duration, time to objective response (TOR), overall survival (OS), and progression-free survival (PFS). For patients without disease progression at the time of analysis, the date of the last follow-up was considered right censored. Log-rank tests were used for comparisons of the Kaplan-Meier survival curves. All tests of hypotheses were conducted at the alpha of 0.05 level with a $95 \%$ CI. ${ }^{16}$ 


\section{Results}

We identified 386 adult patients diagnosed by histopathology and IHC with advanced-stage lung adenocarcinoma treated between January 2013 and September 2017 at both centers. We identified ALK rearrangement in 38 of these using FISH. The median age of the study subjects was 53 years (41-82), with five patients above 65 years and a male predominance (70.8\%). Nonsmokers composed more than half of the patients (57.9\%). The majority of patients (36 patients; 94.7\%) had a good performance status (PS) according to "Eastern Cooperative Oncology Group (ECOG)". One patient had an ECOG PS of 3 due to an extensive lumber vertebral metastasis that needed fixation and postoperative irradiation. He moved with the assistance of a walker or by wheelchair. Another patient had ECOG PS of 4, on mechanical ventilation secondary to respiratory failure, and we initiated crizotinib therapy through a nasogastric tube. ${ }^{17}$

Five patients (13.2\%) had stage IIIB disease, while the rest $(86.8 \%)$ had stage IV disease, of whom 11 patients (28.9\%) were diagnosed with a single-site metastasis. A pretreatment staging workup identified that bones (24.1\%), liver (23.7\%), and brain (21.1\%) were the most common sites for metastases (Table 1). Three patients (7.9\%) were known to have NSCLC after lobectomy for early-stage disease. At follow-up, these patients had developed distant metastases. Biopsies from the metastatic sites confirmed ALK-positive disease.

\section{Treatment received, treatment interruptions, and dose modification}

More than half of the patients $(55.3 \%)$ were treated with a platinum-based combination at some point before crizotinib initiation, either as a postoperative adjuvant therapy or as a palliative therapy for a metastatic disease before the availability of the ALK testing results. A wide variety of platinum-based combinations were used: pemetrexed, paclitaxel, gemcitabine, or vinorelbine plus a platinum component with/without bevacizumab. Before the initiation of crizotinib, seven patients (18.4\%) received palliative RT either as a whole brain irradiation for multiple brain metastases, for palliation of tender bony metastasis, or as a hemostatic dose. Three patients $(7.9 \%)$ were treated for solitary brain metastasis by gamma knife brain surgery.

On the initiation of crizotinib, the majority of patients ( 32 patients; $84.2 \%$ ) tolerated the $250 \mathrm{mg}$ oral (PO)/BID dose with no significant interruptions. Treatment interruptions and dose modification were recommended by the treating
Table I Patient characteristics at baseline $(n=38)$

\begin{tabular}{|c|c|}
\hline Parameter & $\mathbf{N}(\%)$ \\
\hline Median age (range), years & $53(4 \mid-82)$ \\
\hline \multicolumn{2}{|l|}{ Gender, n (\%) } \\
\hline Male & $25(65.8)$ \\
\hline Female & $13(34.2)$ \\
\hline \multicolumn{2}{|l|}{ Race } \\
\hline Arab & $28(73.7)$ \\
\hline Non-Arab & $10(26.3)$ \\
\hline \multicolumn{2}{|l|}{ Smoking status } \\
\hline Current or former smoker & $10(26.3)$ \\
\hline Never smoker & $22(57.9)$ \\
\hline Ex-smoker & $6(15.8)$ \\
\hline \multicolumn{2}{|l|}{ Comorbidities } \\
\hline Hypertension & $15(39.5)$ \\
\hline Diabetes & $16(42.1)$ \\
\hline Cardiac & $6(15.8)$ \\
\hline Renal impairment & $5(13.2)$ \\
\hline Obese $\left(B M I \geq 30 \mathrm{~kg} / \mathrm{m}^{2}\right)$ & $4(10.5)$ \\
\hline \multicolumn{2}{|l|}{ ECOG PS } \\
\hline 0 & $10(26.3)$ \\
\hline I & $21(55.3)$ \\
\hline 2 & $5(13.2)$ \\
\hline 3 & I (2.6) \\
\hline 4 & $\mathrm{I}(2.6)$ \\
\hline \multicolumn{2}{|l|}{ Major symptoms } \\
\hline Shortness of breath & $24(63.2)$ \\
\hline Weight loss & $18(47.4)$ \\
\hline Pain & $9(23.7)$ \\
\hline Headache & $5(13.2)$ \\
\hline \multicolumn{2}{|l|}{ TNM stage } \\
\hline Stage IIIB & $5(13.2)$ \\
\hline Stage IV & $33(86.8)$ \\
\hline \multicolumn{2}{|l|}{ Number of metastatic sites } \\
\hline Multiple sites & $22(57.9)$ \\
\hline Single & II (28.9) \\
\hline \multicolumn{2}{|l|}{ Sites of metastasis } \\
\hline Bone & $16(24.1)$ \\
\hline Liver & $9(23.7)$ \\
\hline Brain & $8(21.1)$ \\
\hline Abdominal lymph nodes & $8(2 \mathrm{I} .1)$ \\
\hline Adrenal(s) & $7(18.4)$ \\
\hline Contralateral lungs & $4(10.5)$ \\
\hline
\end{tabular}

Abbreviations: BMI, body mass index; ECOG, Eastern Cooperative Oncology Group; PS, performance status.

physician for six patients (15.8\%) because of intolerable adverse events (Table 2).

Failure of crizotinib was reported in 16 patients (42.1\%), of whom 15 patients had clinical and radiological disease progression on crizotinib. During crizotinib therapy, intracranial relapse with a limited number of brain lesions occurred in four patients. These patients had good extracranial disease control and continued on crizotinib therapy after controlling the intracranial relapse; two patients (12.5\%) received gamma 
Table 2 Treatment received, interruptions, and dose modifications

\begin{tabular}{|l|l|l|}
\hline \multicolumn{2}{|l|}{ Parameter } & N (\%) \\
\hline Prior therapy & Platinum-based chemotherapy & 2 I (55.3) \\
& RT & $7(18.4)$ \\
& Primary tumor resection & $3(7.9)$ \\
& Gamma knife brain therapy & $3(7.9)$ \\
\hline $\begin{array}{l}\text { Concurrent } \\
\text { therapy }\end{array}$ & RT & $5(13.2)$ \\
& Gamma knife brain therapy & $2(5.3)$ \\
& Craniotomy & I (2.6) \\
\hline Crizotinib timing & Stereotactic brain surgery & I (2.6) \\
& First line & $20(52.6)$ \\
\hline Crizotinib dosage & Subsequent line & $18(47.4)$ \\
\hline Treatment & 250 mg twice daily & $32(84.2)$ \\
interruptions & 200 mg twice daily & $6(15.8)$ \\
\hline Line of treatment & Yes & $8(21.1)$ \\
after failure on & No & $30(78.9)$ \\
crizotinib & Chother TKI & $9(60.0)$ \\
\hline
\end{tabular}

Abbreviations: BSC, best supportive care; RT, radiotherapy; TKI, tyrosine kinase inhibitor.

knife brain therapy, one patient $(6.3 \%)$ received stereotactic brain surgery, and another patient underwent a craniotomy (Table 3A).

Adverse events were recorded in 38 patients and are summarized in Table $3 \mathrm{~B}$. The most commonly reported events were fatigue (68.4\%), peripheral edema $(63.2 \%)$, and transaminitis (50\%). Grade III transaminitis occurred in two patients. After a few days of treatment, enzyme titers returned to basal levels and crizotinib was resumed and continued at a lower dosage ( $200 \mathrm{mg}$ orally twice daily) without further interruptions. Grade IV transaminitis was reported in another patient. After normalization of the hepatic enzymes, this patient was changed to alectinib treatment, but unfortunately, the same adverse event was reported. Finally, this patient commenced chemotherapy and showed a good tolerance to it.

Diarrhea (grade III/IV) occurred in three of the cases (7.9\%), leading to dehydration and a slight elevation of blood urea nitrogen in one patient. Uncomplicated neutropenia was reported in six patients $(15.8 \%)$.

Bradycardia with prolonged QT interval occurred in 10 patients $(26.3 \%)$. Three were grade III/IV. One of these patients was a 72-year-old female, who was morbidly obese, with a cardiac pacemaker implanted for the previous 7 years. On day 9 of crizotinib usage, she fainted and was found to have severe sinus bradycardia (heart rate was 30 beats/ min) and significant prolongation of the QT interval. She was admitted to a cardiac care unit where crizotinib was
Table 3 Crizotinib failure and adverse events (38 patients)

\begin{tabular}{|l|l|l|}
\hline \multicolumn{2}{|l|}{ (A) Crizotinib failure (16 patients) } & N (\%) \\
\hline Parameter & Oligo metastasis & $2(12.5)$ \\
\hline \multirow{3}{*}{ Pattern of failure } & Multiple metastasis only & $6(37.5)$ \\
\cline { 2 - 3 } & Locoregional only & $3(18.7)$ \\
\cline { 2 - 3 } & $\begin{array}{l}\text { Locoregional and distant } \\
\text { metastasis }\end{array}$ & $4(25.0)$ \\
\cline { 2 - 3 } & Poor tolerance to treatment & I (6.3) \\
\hline Intracranial relapse & \multicolumn{2}{|l|}{} \\
\hline (B) Crizotinib adverse events (38 patients) & \\
\hline Parameter & Any grade & Grade III/IV \\
\hline Fatigue & $26(68.4)$ & I (2.6) \\
\hline Peripheral edema & $24(63.2)$ & $2(5.3)$ \\
\hline Transaminitis & $19(50.0)$ & $3(7.9)$ \\
\hline Skin changes & $14(36.8)$ & $0(0.0)$ \\
\hline Diarrhea & $12(32.6)$ & $3(7.9)$ \\
\hline Bradycardia & $11(28.9)$ & $0(0.0)$ \\
\hline Prolonged QT & $10(26.3)$ & $3(7.9)$ \\
\hline Colored vision & $8(2 I .1)$ & $0(0.0)$ \\
\hline Nausea/vomiting & $7(18.4)$ & $0(0.0)$ \\
\hline Neutropenia & $6(15.8)$ & $0(0.0)$ \\
\hline Neuropathy & $6(15.8)$ & $0(0.0)$ \\
\hline Constipation & $3(7.9)$ & $0(0.0)$ \\
\hline & & \\
\hline
\end{tabular}

immediately stopped. After 3 days of monitoring, her heart rate returned to her baseline level. Because of her advanced age, multiple comorbidities, and her advanced cancer with expected poor tolerance to chemotherapy, our thoracic multidisciplinary team and her cardiologist adjusted the pacemaker to a higher rate and resumed crizotinib at $250 \mathrm{mg}$ PO BID. Another two patients, with unknown history of cardiac disease, developed sinus bradycardia and prolongation of the QT interval. They showed good tolerance on the lower dosage.

Of the 38 patients included in efficacy analyses, 36 were suitable for response evaluation (two patients died early from the disease). The objective response rate (ORR) was $50 \%$. Two cases $(5.3 \%)$ were with a complete response (CR), and 17 cases $(44.7 \%)$ were with a partial response (PR). Two cases $(5.3 \%)$ achieved stable disease (SD), while progressive disease (PD) occurred in four cases (16.7\%). The median time to an objective response in 19 patients was 3.4 months (range 2.2-5.2 months, 95\% CI: 3.2-3.7). The time of the response was 19.9 months.

The 1-year survival rate was $88.6 \%$, while 5-year survival rate was $34.8 \%$. The mean PFS was $17.678 \pm 4.227$ months (rang: 9.393- 25.963). Patients with first -line crizotinib had a lower mean PFS of $14.834 \pm 6.789$ months (range: 1.527-28.141) when compared to the patients who received crizotinib as a subsequent line of treatment with PFS of 20.928 \pm 4.958 months (range: $11.210-30.646$ ) with no statistically significant outcome $(P=0.487)$. 
The mean OS was 50.097 \pm 6.547 months (rang: 37.265-62.928 months). Patients with first-line crizotinib treatment had longer mean OS of $48.794 \pm 8.941$ months (rang: 31.270-66.319 months) when compared to patients who received crizotinib as subsequent lines with mean OS of 46.790 \pm 7.827 months (range: 48.794-62.131) with no statistically significant outcome $(\mathrm{P}=0.456)$ (Table 4 and Figures 1 and 2).

\section{Discussion}

Worldwide, lung cancer has been described as the highest malignancy as per public health view. In Kuwait, lung cancer is the sixth most frequent cancer $(6.2 \%$ of all malignancies, the fifth most common in males and the ninth in females). Lung cancer is the most common reason for malignancyrelated mortality (15.9\%) in Kuwaiti males, while among Kuwaiti females, it is the fifth most common reason for malignancy-related mortality (4.9\%). In Saudi Arabia, lung cancer is the sixth most frequent cancer $(4.7 \%$ for all malignancies). In Saudi males, lung cancer is the fourth highest malignancy $(7.4 \%)$ and the second most common cause of malignancy-related mortality (11.2\%). Lung cancer in Saudi females is very rare $(2.2 \%)$, and it is the eighth most common cause of malignancy-related mortality $(4.4 \%) .^{18}$

The OS is the most consistent end point in estimating any investigational new treatment for cancer, with significant progress in OS needed for getting regulatory approval. Post approval, local research is important as well to assess the drug's effectiveness and tolerability.

We performed a retrospective study on the management of cases confirmed as advanced-stage ALK-positive NSCLC. This study used existing data available in the medical charts and electronic medical records of two of the biggest tertiary referral centers for cancer management on the western coast

Table 4 Response and survival outcome

\begin{tabular}{|l|l|l|l|}
\hline Parameter & Evaluated patients & $\mathbf{N}$ & $\%$ \\
\hline Response & $\mathbf{3 6}$ & $\mathbf{9 4 . 7 \%}$ \\
& CR & 2 & $5.3 \%$ \\
& PR & 17 & $44.7 \%$ \\
& SD & 2 & $5.3 \%$ \\
& PD & 15 & $39.5 \%$ \\
\cline { 2 - 4 } & Nonevaluated & 2 (5.3\%) \\
\cline { 2 - 4 } & Median TOR & $\mathbf{3 . 4}$ months \\
& & (range: 2.2-5.2) \\
& & $(\mathbf{9 5 \%}$ Cl: 3.2-3.7) \\
\hline Survival & I-year survival & $\mathbf{8 8 . 6 \%}$ \\
& 5-year survival & $\mathbf{3 4 . 8 \%}$ \\
\hline
\end{tabular}

Abbreviations: $C R$, complete response; PD, progressive disease; PR, partial response; SD, stable disease; TOR, time to objective response. of the Arabian Gulf, the KCCC and the KFSH, from January 2013 to September 2017.

Crizotinib is a first-generation ALK-TKI that has previously been shown to have remarkable activity for the treatment for ALK-positive NSCLC, considering the response rate and PFS results. In August 2011, crizotinib was approved by the FDA for the management of ALK-positive advancedstage NSCLC based on the outcomes of the PROFILE 1014 trial, where crizotinib showed superiority to the first pemetrexed/platinum combination in cases with advanced ALK-positive NSCLC not previously treated. Treatment with crizotinib resulted in longer PFS, higher ORR, and more tolerable adverse events than chemotherapy. ${ }^{19}$ Although there were fewer subjects in our study than in the PROFILE 1014 trial (38 vs 172 subjects), there were some differences. We detected ALK rearrangement in $9.8 \%$ of cases of lung adenocarcinoma, while in most of the published studies, ALK rearrangement was reported in $5-7 \%$ of cases. ${ }^{4,5}$ Most of the subjects in our study were young, Asian, and nonsmoker females, and this may explain the high prevalence of ALKpositive NSCLC in our study. The median age of our cases was 53 years, and most of these cases were nonsmokers $(57.9 \%)$.

Similar results were shown in the PROFILE 1014 trial (age 52 years, and $65 \%$ nonsmokers). Because of the toxicity profiles of all the approved ALK-TKIs used in clinical practice, we were able to administer crizotinib via nasogastric tube in a patient with poor PS (ECOG PS 3-4). ${ }^{17}$

At the time of initial diagnosis, central nervous system (CNS) metastasis was reported in $21.1 \%$ of our patients vs $38 \%$ in the PROFILE 1014 trial. The strategy we applied for controlling CNS disease is supported by the results of this trial, including the effects of whole-brain irradiation, gamma knife brain surgery, stereotactic brain surgery, craniotomy, and following up of asymptomatic small brain lesions. ${ }^{20}$

In the PROFILE 1014 trial, the ORR for crizotinib was $74 \%$. The median PFS was extended more with crizotinib than with chemotherapy " 10.9 vs 7.0 months". The median OS was not reached by crizotinib; the likelihood of 1-year survival with crizotinib was stable at $84 \%$. In our study, the ORR for crizotinib was $50 \%$, and the median PFS was 16.5 months (95\% CI: 1.6-31.4). The median OS from the initiation of crizotinib was 48.0 months (95\% CI: 18.2-77.8). In the near future, ALK mutation analysis may play a role in explaining why some ALK-positive NSCLC patients do not respond well to crizotinib. ${ }^{21}$

When the use of standard chemotherapeutic agents results in clinical failure, it is feasible to switch to crizotinib as per 

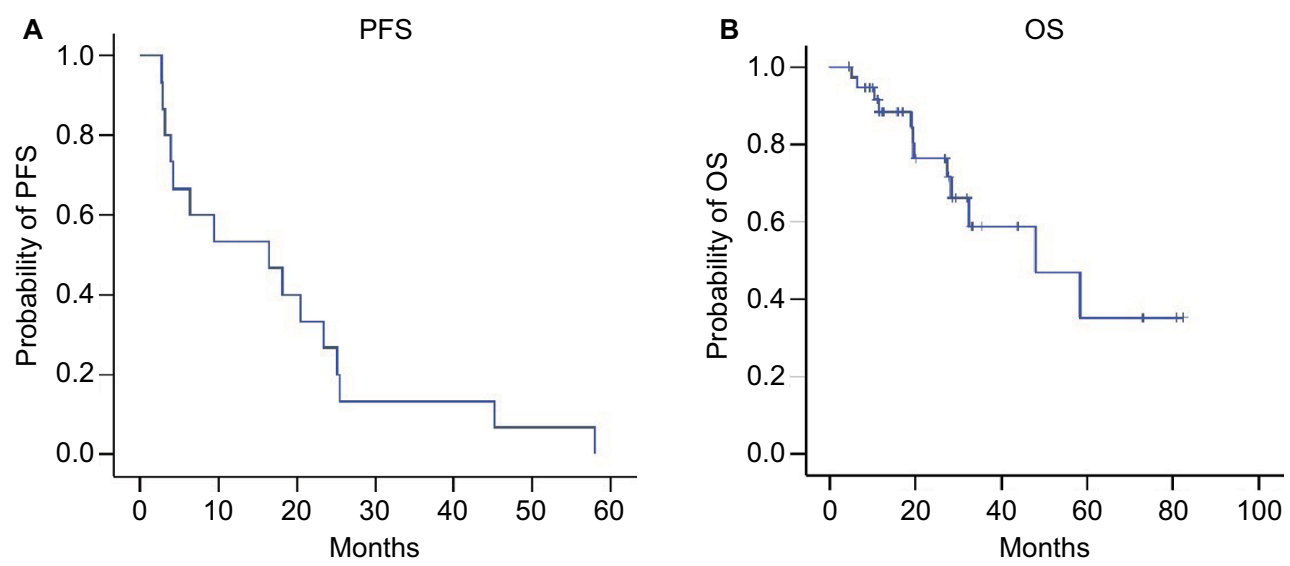

Figure I Kaplan-Meier estimates of survival functions in intention-to-treat population.

Notes: (A) The mean PFS was 17.678 \pm 4.227 months (range: $9.393-25.963$ ). (B) The I-year survival was $88.6 \%$ and 5 -year survival as $34.8 \%$.The mean OS was $50.097 \pm 6.547$ months (rang: 37.265-62.928).

Abbreviations: OS, overall survival; PFS, progression-free survival.
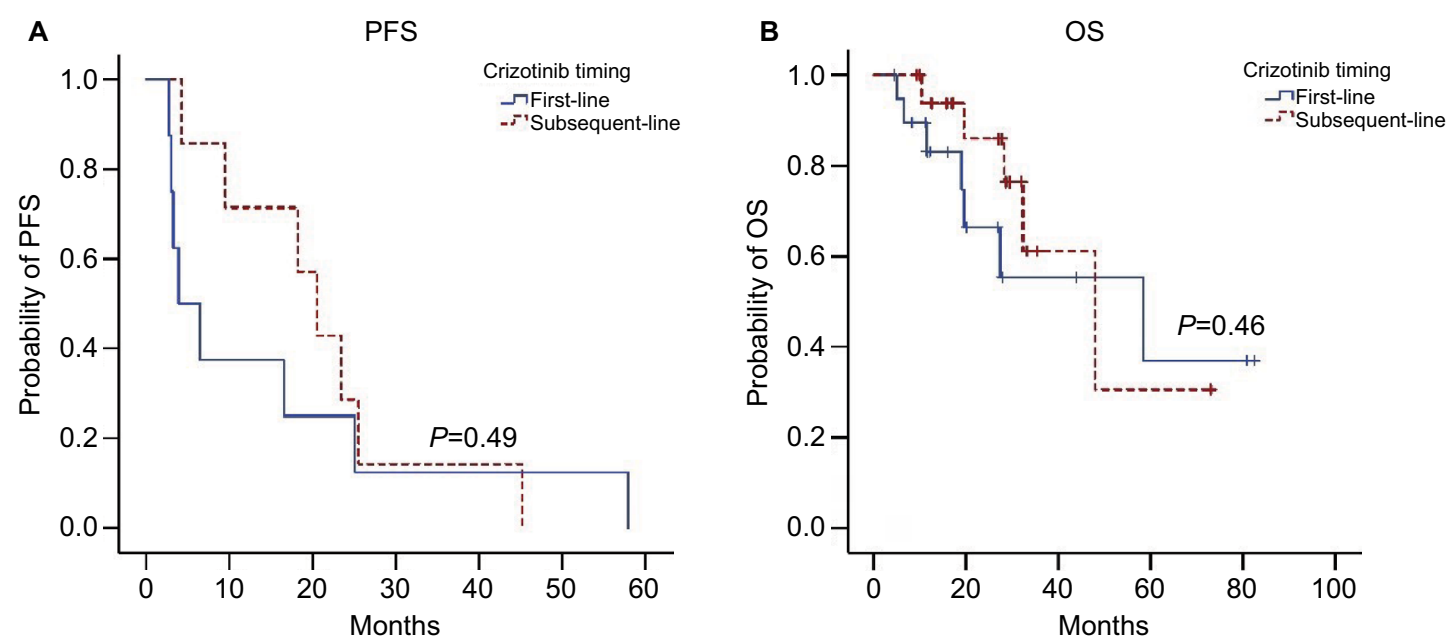

Figure 2 Kaplan-Meier estimates of survival functions stratified by prior platinum-based chemotherapy in intention-to-treat population.

Notes: (A) Patients with first line crizotinib had less mean PFS of 14.834 \pm 6.789 months (range: 1.527-28.14I) and patients received crizotinib as subsequent lines with PFS of $20.928 \pm 4.958$ months with no statistically significant outcome ( $P=0.487)$. (B) Patients with first line crizotinib had longer mean OS of $48.794 \pm 8.94 I$ months and patients received crizotinib as subsequent lines with OS of $46.790 \pm 7.827$ months with no statistically significant outcome $(P=0.456)$.

Abbreviations: OS, overall survival; PFS, progression-free survival.

prior first- and second-line registration trials of crizotinib. In our study, the survival differences between the cases that received standard chemotherapy before and chemotherapynaive cases were challenging based on the confounding factors involved in patient selection and previous chemotherapy exposure. Our analysis revealed no substantial difference in PFS ( $P$-value was 0.49$)$ or OS ( $P$-value was 0.46$)$ between cases exposed to standard platinum-based combination chemotherapy before crizotinib and those who received crizotinib as the first-line treatment. These differences could be elucidated in a randomized controlled study. ${ }^{22}$

Lee et $\mathrm{al}^{23}$ concluded in their study that ALK positivity was unconventionally predictive of pemetrexed effectiveness in NSCLC cases. ALK-positive crizotinib-naive patients have a significantly longer PFS when receiving pemetrexed.

\section{Conclusion}

Crizotinib seems to be a promising agent for the management of advanced ALK-positive NSCLC. Crizotinib had a good response rate, a very acceptable side effect profile, promising median PFS, and median OS.

\section{Ethical approval}

This study did not entail any experimental procedures or treatments of human participants by any of the authors. The research ethics committees of the Sheikha Badriya Alsabah Center, KCCC, Kuwait, and KFSH, Saudi Arabia, approved this study. Given the retrospective nature of this study and its use of deidentified data, a waiver for obtaining individual patient consent was requested and approved by the ethics committees. All patient data were stored safely, securely 
coded, and de-identified to ensure confidentiality with limited access by the corresponding author.

\section{Acknowledgment}

The abstract of this paper was presented at the ASCO Annual Meeting, USA (June 2017), as a poster presentation with interim findings. The poster's abstract was published in "Poster Abstracts" in the Journal of Clinical Oncology 35, no. 15_suppl, DOI: 10.1200/JCO.2017.35.15_suppl.e20516 https://meetinglibrary.asco.org/record/146588/abstract.

\section{Disclosure}

The authors report no conflicts of interest in this work.

\section{References}

1. American Cancer Society's Cancer Statistics Center [webpage on the Internet]. Key Statistics for Lung Cancer; 2018. Available https://www. cancer.org/cancer/non-small-cell-lung-cancer/about/key-statistics.html. Accessed October 16, 2018.

2. Kohno T, Nakaoku T, Tsuta K, et al. Beyond ALK-RET, ROS1 and other oncogene fusions in lung cancer. Transl Lung Cancer Res. 2015; 4(2): 156 .

3. Li T, Kung HJ, Mack PC, Gandara DR. Genotyping and genomic profiling of non-small-cell lung cancer: implications for current and future therapies. J Clin Oncol. 2013;31(8):1039-1049.

4. Camidge DR, Doebele RC. Treating ALK-positive lung cancer-early successes and future challenges. Nat Rev Clin Oncol. 2012;9(5):268-277.

5. Soda M, Choi YL, Enomoto M, et al. Identification of the transforming EML4-ALK fusion gene in non-small-cell lung cancer. Nature. 2007;448(7153):561-566.

6. Morris SW, Kirstein MN, Valentine MB, et al. Fusion of a kinase gene, ALK, to a nucleolar protein gene, NPM, in non-Hodgkin's lymphoma. Science. 1994;263(5151):1281-1284.

7. Chen Y, Takita J, Choi YL, et al. Oncogenic mutations of ALK kinase in neuroblastoma. Nature. 2008;455(7215):971-974.

8. Coffin CM, Patel A, Perkins S, Elenitoba-Johnson KS, Perlman E, Griffin CA. ALK1 and p80 expression and chromosomal rearrangements involving $2 \mathrm{p} 23$ in inflammatory myofibroblastic tumor. Mod Pathol. 2001;14(6):569-576.
9. Christensen JG, Zou HY, Arango ME, et al. Cytoreductive antitumor activity of PF-2341066, a novel inhibitor of anaplastic lymphoma kinase and c-Met, in experimental models of anaplastic large-cell lymphoma. Mol Cancer Ther. 2007;6(12 Pt 1):3314-3322.

10. Kazandjian D, Blumenthal GM, Luo L, et al. Benefit-risk summary of crizotinib for the treatment of patients with ROS1 alteration-positive, metastatic non-small cell lung cancer. Oncologist. 2016;21(8):974-980.

11. Khozin S, Blumenthal GM, Zhang L, et al. FDA approval: ceritinib for the treatment of metastatic anaplastic lymphoma kinase-positive non-small cell lung cancer. Clin Cancer Res. 2015;21(11):2436-2439.

12. Larkins E, Blumenthal GM, Chen H, et al. FDA Approval: Alectinib for the Treatment of Metastatic, ALK-Positive Non-Small Cell Lung Cancer Following Crizotinib. Clin Cancer Res. 2016;22(21):5171-5176.

13. Markham A. Brigatinib: First Global Approval. Drugs. 2017;77(10): $1131-1135$.

14. Peters S, Camidge DR, Shaw AT, et al. Alectinib versus Crizotinib in Untreated ALK-Positive Non-Small-Cell Lung Cancer. N Engl J Med. 2017;377(9):829-838.

15. Shaw AT, Friboulet L, Leshchiner I, et al. Resensitization to Crizotinib by the Lorlatinib ALK Resistance Mutation L1198F. N Engl J Med. 2016;374(1):54-61.

16. Pazdur R. Endpoints for assessing drug activity in clinical trials. Oncologist. 2008;13(Suppl 2):19-21.

17. Tamai K, Nagata K, Otsuka K, et al. Crizotinib administered via nasogastric and percutaneous endoscopic gastrostomy tubes for the successful treatment of ALK-rearranged lung cancer in a patient with poor performance status. Respir Investig. 2013;51(1):46-48.

18. Ferlay J, Soerjomataram I, Dikshit R, et al. Cancer incidence and mortality worldwide: sources, methods and major patterns in GLOBOCAN 2012. Int J Cancer. 2015 ;136(5):E359-86.

19. Solomon BJ, Mok T, Kim DW, et al. First-line crizotinib versus chemotherapy in ALK-positive lung cancer. $N$ Engl J Med. 2014;371(23): 2167-2177.

20. Bruynzeel AM, Lagerwaard FJ. Whole brain radiotherapy for brain metastases from non-small cell lung cancer: the end of an era? JThorac Dis. 2016;8(11):E1525-E1527.

21. Doebele RC, Pilling AB, Aisner DL, et al. Mechanisms of resistance to crizotinib in patients with ALK gene rearranged non-small cell lung cancer. Clin Cancer Res. 2012;18(5):1472-1482.

22. Shaw AT, Yeap BY, Solomon BJ, et al. Effect of crizotinib on overall survival in patients with advanced non-small-cell lung cancer harbouring ALK gene rearrangement: a retrospective analysis. Lancet Oncol. 2011;12(11):1004-1012.

23. Lee JO, Kim TM, Lee SH, et al. Anaplastic lymphoma kinase translocation: a predictive biomarker of pemetrexed in patients with non-small cell lung cancer. J Thorac Oncol. 2011;6(9):1474-1480.
Cancer Management and Research

\section{Publish your work in this journal}

Cancer Management and Research is an international, peer-reviewed open access journal focusing on cancer research and the optimal use of preventative and integrated treatment interventions to achieve improved outcomes, enhanced survival and quality of life for the cancer patient. The manuscript management system is completely online and includes

\section{Dovepress}

a very quick and fair peer-review system, which is all easy to use. Visit http://www.dovepress.com/testimonials.php to read real quotes from published authors. 